\title{
REVISÃO DE LITERATURA E EVIDENCIAS EMPÍRICAS SOBRE ECONOMIA DO CRIME
}

\author{
Cleiton Franco ${ }^{1}$
}

\begin{abstract}
Resumo
O objetivo deste trabalho é realizar uma revisão de literatura relacionada a economia do crime no Brasil. A revisão da literatura a partir do artigo seminal de Becker evidencia que há muito o que explorar a respeito da teoria do crime, principalmente no Brasil. Os resultados apontam que a ampliação da discussão foi possível graças a contribuições nos campos da dissuasão (deterrence) e de características locais (bairro, salários, vizinhança) do individuo, proposta por Glaeser e Sacerdote (1999). As principais evidências empíricas apontam contribuições no campo da criminalidade e violência urbana associada à discussão envolvendo crescimento econômico, desigualdade de renda, e o agente governamental na melhoria das politicas públicas.
\end{abstract}

Palavras-chave: economia do crime; produção científica; evidências empíricas; Brasil.

\section{INTRODUÇÃO}

A partir da década de 1960, surgiram as primeiras contribuições à ciência econômica no debate sobre teoria do crime. As mais diversas variáveis socioeconômicas têm sido incorporadas a investigação empírica do crime: renda, taxa de desemprego, nível de escolaridade, pobreza, desigualdade de renda e urbanização, desde os trabalhos de Fleisher (1963; 1966), de Becker (1968) e Ehrlich (1973). A fundamentação teórica da racionalidade econômica do crime foi aprofundada por Becker (1968). Seu trabalho propunha o entendimento de que decisões de cometer um crime decorrem de avaliações racionais de possíveis ganhos e de outra forma, os custos associados ao ato da transgressão. Desta maneira, a mentalidade criminosa também estaria sujeita ao trade-off que afeta todos os demais agentes racionais: a alocação ótima do tempo nas varias atividades, divididas entre lícitas e ilícitas. A maximização da utilidade apresentará por meio da utilidade esperada a decisão de transgredir o ordenamento jurídico estabelecido e o resultado desse processo.

Os custos da criminalidade são representativamente altos para a sociedade e para o Estado, e incorporam gastos materiais, gastos públicos e privados, tanto na prevenção quanto no combate ao crime. Segundo Cerqueira et al (2007) apenas no período de 2004, o custo da violência no Brasil foi de $\mathrm{R}$ \$ 92,2 bilhões, o que representou cerca de 5,09\% do PIB. Deste total, R\$ 28,7 bilhões corresponderam a despesas efetuadas pelo setor público e R $\$ 60,3$ bilhões foram associados aos custos tangíveis e intangíveis arcados pelo setor privado. Os efeitos gerados pela criminalidade afetam o bem estar e reduzem a qualidade de vida nas

\footnotetext{
${ }^{1}$ UNEMAT 
grandes cidades, além do efeito negativo sobre o estoque de capital humano, como estimado por Carvalho et al (2007) o prejuízo montante derivado das mortes por homicídios de nove bilhões de reais.

A segurança pública converteu-se num dos maiores itens do orçamento e objeto de discussão prioritária. Pesquisas de opinião pública revelam que, ao lado do desemprego, a violência aparece entre as maiores causas da inquietação popular. Os gastos com segurança pública no período de 1995 a 2005 comprometeram em média 1,34\% do PIB do país. A despesa média per capta, ou quanto custou à violência por ano por brasileiro, foi de $\mathrm{R} \$$ 144,88 no período analisado (TABELA 1 ).

\section{Tabela 1 - Estimativa dos custos com segurança pública no Brasil (1995 A 2005) em} milhões de R\$

\begin{tabular}{|c|c|c|c|c|c|c|}
\hline Ano & Municípios & Estados & União & Custo total & Relação custo/PIB & Custo per capita \\
\hline 1995 & 705 & 15510 & 3788 & 20003 & $1,16 \%$ & 127,63 \\
\hline 1996 & 721 & 16446 & 3737 & 20904 & $1,10 \%$ & 131,22 \\
\hline 1997 & 734 & 17947 & 3835 & 22516 & $1,13 \%$ & 139,03 \\
\hline 1998 & 861 & 20116 & 3902 & 24879 & $1,21 \%$ & 151,12 \\
\hline 1999 & 771 & 18390 & 3368 & 22529 & $1,24 \%$ & 134,61 \\
\hline 2000 & 722 & 20587 & 3737 & 25047 & $1,33 \%$ & 147,21 \\
\hline 2001 & 767 & 24645 & 4034 & 29446 & $1,59 \%$ & 170,82 \\
\hline 2002 & 818 & 23059 & 2673 & 26550 & $1,63 \%$ & 152,03 \\
\hline 2003 & 920 & 22131 & 2729 & 25780 & $1,46 \%$ & 145,75 \\
\hline 2004 & 1158 & 21003 & 2807 & 24968 & $1,39 \%$ & 139,4 \\
\hline 2005 & 1232 & 23836 & 3018 & 28087 & $1,45 \%$ & 154,89 \\
\hline Média do Período & 855,36 & $20.333,64$ & $3.420,73$ & $24.609,91$ & $1,34 \%$ & 144,88 \\
\hline Var. Média anual & $5,70 \%$ & $4,40 \%$ & $-2,20 \%$ & $3,50 \%$ & $2,30 \%$ & $2 \%$ \\
\hline
\end{tabular}

Fonte: secretaria do tesouro nacional-stn (dados brutos); IBGE-contas nacionais e estimativas populacionais (dados brutos); IPEA-grupo de estudos da violência (cálculos e derivações).

Os gastos com segurança pública afetam os gastos com saúde. Em 2004 esses gastos representaram cerca de 4 bilhões de reais. Na segurança privada, entre 2000 e 2005 cresceram a uma taxa anual de 3,2\%. Em 2005, o custo total era de $\mathrm{R} \$ 14,4$ bilhões e correspondia a 0,79\% do PIB, representando um gasto per capita da ordem de $\mathrm{R} \$ 81,93$. Em relação ao setor de seguros privados no Brasil, com base em informações do Ministério da Fazenda e Superintendência de Seguros Privados (Susep/Decon/Geest), no período entre 1995 e 2005, os gastos com seguros apresentaram um crescimento real anual na ordem de 1,39\%. Em 2005 a sociedade havia despendido com seguros cerca de 14,5 bilhões. A alocação de recursos para essas modalidades de seguros correspondeu a $0,75 \%$ do PIB (TABELA 2).

Tabela 2 - Custos sociais da violência no Brasil em 2004 


\begin{tabular}{lrrr}
\hline Setor público & \multicolumn{1}{c}{ total } & \% PIB & \multicolumn{2}{c}{ per capita } \\
Segurança pública & $28.087,00$ & $1,45 \%$ & 154,89 \\
Sistema prisional & $2.814,00$ & $0,15 \%$ & 15,52 \\
Sistema de saúde & 988,00 & $0,06 \%$ & 5,44 \\
Custo total público & $31.889,00$ & $1,65 \%$ & 175,85 \\
\hline Setor privado & total & $\%$ PIB & per capita \\
Perda Capital humano & $23.868,00$ & $1,35 \%$ & 131,44 \\
Segurança privada & $14.317,00$ & $0,80 \%$ & 79,94 \\
Seguros & $12.709,00$ & $0,75 \%$ & 80,3 \\
Transferencias roubos/furtos & $9.419,00$ & $0,53 \%$ & 51,87 \\
Custo total Privado & $60.313,00$ & $3,43 \%$ & 343,55 \\
Custo Social & $92.202,00$ & $5,09 \%$ & 519,4 \\
\hline
\end{tabular}

Fonte: grupo de estudos da violência do IPEA.

As contribuições à teoria do crime, no tocante aos últimos 30 anos, tem se multiplicado ao nível internacional, por meio de pesquisas com dados de cross section e/ou séries temporais (WOLPIN, 1978; CRAIG, 1987; TRUMBULL, 1989; ZHANG, 1997, entre outros) ou através de estimações com dados em painel (WOLPIN, 1980; CORNWELL e TRUMBULL, 1994; FAJNZYLBER, EDERMANE e LOYZA, 1998; GOULD, WEINBERG e MUSTARD, 2000, entre outros.). No Brasil, merece destaque os trabalhos empíricos de Kume (2004), Oliveira (2005), Hartung e Pessoa (2007), Loureiro e Carvalho Júnior (2007), Santos e Kassouf (2007). No trabalho de Hartung e Pessoa (2007) os modelos incorporaram variáveis demográficas. Mais recentemente, os trabalhos consideraram a relação entre legalização do aborto e redução da criminalidade, vinte anos depois, nos Estados Unidos (Donohue e Levitt, 2001; Levitt, 2004).

O objetivo deste trabalho é realizar uma revisão de literatura da produção cientifica relacionada a economia do crime no Brasil. A justificativa para uma revisão da literatura da produção cientifica no Brasil a respeito da teoria do crime é agregar as contribuições encontradas na literatura. Busca-se, evidenciar as causas da criminalidade e os efeitos do comportamento do individuo criminoso no Brasil.

O trabalho está estruturado da seguinte forma: na seção 2, apresenta-se o referencial teórico a respeito da economia do crime, desde o trabalho seminal de Becker e seu modelo inicial, onde avalia-se a racionalidade do agente criminoso, passando por Glaeser (1999) e a exploração da criminalidade e o tamanho das cidades. Na seção 3 e 4 são apresentadas as evidências e contribuições científicas a partir da reunião dos trabalhos sobre economia do crime no Brasil, violência urbana e alocação da força policial. 


\section{A ECONOMIA DO CRIME E A EVOLUÇÃO DO MODELO TEÓRICO DE BECKER}

A teoria do crime, proposta por Becker (1968) propõe a humanização do individuo criminoso, que deixa de ser apenas um sujeito com desvios de personalidade e propensão a má conduta e passa a ser avaliado como um indivíduo comum, incentivado por impulsos racionais e, em termos de possibilidade de maximizar sua utilidade através de ganhos e ponderação de custos associados a atividade ilegal. Seguindo a ideia proposta por Becker, autores como Sjoquist (1973), Ehrlich (1973) e Block e Heinecke (1975) dedicaram-se a expandir os estudos econômicos do crime propondo análise dos efeitos de deterrence (efeitos dissuasórios) que afetam o comportamento criminoso e na identificação de caracteristicas observáveis (como salário, posição social, distribuição de renda, entre outros) que poderiam levar o indivíduo a atividade criminosa.

Os pressupostos básicos do modelo levam em consideração a probabilidade de prisão, ganhos e perdas associadas a custos para o individuo e sociedade na atividade ilícita, e o fator da renda alternativa na atividade criminosa, onde todos os agentes possuem as mesmas habilidades (BRUECKNER, 2011).

a representa a probabilidade de ser preso, que depende das características da cidade (ex. da densidade, da iluminação pública, do policiamento, etc.);

$L$ representa o ganho com a atividade criminosa; assim, o ganho esperado desta atividade é dado por:

$$
E(L)=a \times 0+(1-a) \times L=(1-a) \cdot L
$$

$j$ representa os custos para o indivíduo no caso de ser capturado, que também depende das características da cidade (ex. tamanho da pena, funcionamento do sistema jurídico, etc.); assim, os custos esperados associados à captura são dados por:

$$
E(j)=a \times j+(1-a) \times 0=a \cdot j
$$

$e$ representa os custos da atividade criminosa (ex. acesso a armamento, planejamento, etc.), que também depende das características da cidade (ex. policiamento, leis de tolerância de acesso a armas, etc.) Com tais hipóteses, a renda esperada da atividade criminosa pode ser representada por:

$$
E(L)-E(j)-e \quad \text { ou } \quad(1-a) \cdot L-a . j-e
$$

Desta forma, o crime seria cometido quando:

$$
(1-a) . L-a . j-e>\text { ganhos com atividades lícitas na cidade }
$$


Em termos mais científicos de decomposição do modelo, Becker (1968) propõe a existência de uma função que relaciona o número de transgressões (crimes ou infrações) realizadas por um individuo racional $\left(O_{j}\right)$ com probabilidade de condenação $\left(p_{j}\right)$, com punição por transgressão, caso haja condenação $\left(f_{j}\right)$ e com outras variáveis que possam incitá-lo a cometer ilicitudes $\left(u_{j}\right)$. Assim:

$$
O j=O j(p j, f j, u j)
$$

Às variáveis de deterrence $p_{j}$ e $f_{j}$ imputam redução do número de transgressões, de forma $\partial O j / \partial p j<0$, assim como $\partial O j / \partial f j<0$.

De forma complementar, a utilidade líquida esperada do indivíduo $j$ pelo cometimento de uma transgressão $\left(E U_{j}\right)$ é definida por:

$$
E U j=[p j U j(Y j-f j)]+(1-p j) U j(Y j)
$$

Em que $U_{j}$ representa a função utilidade do indivíduo $j$ e $Y_{j}$ é o ganho ou vantagem advinda da transgressão. De forma intuitiva, se a punição pela transgressão $\left(f_{j}\right)$ superar os benefícios da atividade ilícita $\left(Y_{j}\right)$ e a probabilidade de ser condenado for relativamente alta, a utilidade esperada será negativa, o que inviabiliza o ato criminoso. Por outro lado, se a punição $\left(f_{j}\right)$ for pequena ou irrelevante para o indivíduo, reduzida em relação aos benefícios previstos, a transgressão poderá ocorrer ainda diante da alta probabilidade de condenação.

O crime também pode afetar também a sociedade e não apenas o infrator e sua vítima. Becker (1968) interpreta que apresentar o custo social em termos do custo incorrido pelo transgressor facilita as variáveis de escolha ótima para a ação do governo e autoridades. Assim, $f^{\prime} \equiv b f$, ou seja, $f$ além de simbolizar a punição para os condenados também reflete o custo por transgressão, ao passo que $f$ ' representa o custo social e $b$ é o coeficiente que transforma $f$ em $f^{\prime}$. O tamanho de $b$, que nada mais é do que a forma escolhida para a punição, podendo variar de valores próximos a zero (para pequenas infrações, por exemplo) até $b>1$ para confinamentos penitenciários e outros tipos de punição (prisão perpétua, pena de morte, etc.).

Esforços de deterrence $\left(p_{j}\right)$ podem efetivamente reduzir o número de transgressões, porém implicam aumentos no custo social por meio da elevação nos custos totais de combate à criminalidade $(C)$. Corman e Mocan (2000) afirmam que alguns trabalhos evidenciam que aumentos nas atividades de dissuasões reduzem o crime, 
enquanto outros encontram fraca relação entre crime e as variáveis de deterrence. Levitt (1997) estudou os ciclos eleitorais sobre o uso de policiais como instrumento para as variáveis de deterrence. Os resultados sugerem que aumentos na forca policial possuem o efeito de reduzir crimes violentos, porém geram menor impacto sobre crimes contra a propriedade.

Wolpin (1978), que trabalhou com longa série temporal de dados, discutindo seis tipos diferentes de crime ocorridos na Inglaterra e no País de Gales, desde 1894 a 1967, utilizando também seis variáveis diferentes de dissuasão judicial: taxa de esclarecimento do crime, taxa de condenação, taxa de aprisionamento, taxa de multa, taxa de reconhecimento e tempo de sentença média. Dentre essas variáveis dissuasórias, as mais estatisticamente significativas foram a taxa de esclarecimento e a taxa de aprisionamento. Quanto a variável punição, o resultado apresentou estatísticas não-significativas em todas as regressões geradas. Quanto aos efeitos decorrentes do desemprego e de maiores proporções de jovens na população, o estudo captou a relação positivamente significativa, replicando os resultados já encontrados por Ehrlich (1973). Assim, torna-se importante conhecer a função que mensura as perdas sociais advindas das atividades criminais, representada por:

$$
L=L(D, C, b f, O)
$$

em que $D$ aponta os danos sociais, $b f$ (ou $f^{\prime}$ ) representa o custo social por ofensa sofrida e $O$ representa o nível de atividade criminal. Pressupõe-se:

$$
\partial L / \partial D>0, \partial L / \partial C>0, \partial L / \partial b f \text {. }
$$

Becker (1968) assume ainda que a função de perda social é equivalente à função de perda total social em termos da renda real de transgressões, condenações e punições, em que $b p f O$ é a perda social total das punições, uma vez que $b f$ é a perda por transgressão punida e $p O$ representa o número de ofensas punidas, como segue:

$$
L=D(O)+C(p, O)+b p f O
$$

As variáveis que sofrem controle direto dos gestores e representantes da sociedade são os custos de deterrence $(C)$, a punição para os criminosos condenados $(f)$ e a forma como essas punições se darão $(b)$. Uma vez escolhidas essas variáveis, determina-se indiretamente $p, O, D$ e, por consequência, a perda social total $L$. De outra forma, é possível que ações governamentais interfiram nas preferências individuais dos agentes econômicos de forma a permitir uma minimização de $L$ advinda do ato de cometer 
uma quantidade "ótima" de ilícitos.

O modelo de Becker (1968) foi desenvolvido para captar aspectos microeconômicos presentes nos determinantes da criminalidade do individuo. No entanto, os trabalhos posteriormente desenvolvidos, considerando a limitação e/ou inexistência de dados, consolidaram estimativas a partir de informações agregadas em algum nível de regionalização (subdivisões estaduais, no caso brasileiro). Dessa forma, incorre-se num trade-off do tipo maiores unidades geográficas acarretam menor qualidade nas informações médias agregadas, uma vez que menores regionalizações (ou até mesmo individualizações) não são factíveis em termos de análise (CERQUEIRA E LOBÃO, 2003).

As interpretações da racionalidade econômica do comportamento criminoso realizadas numa formalização mais moderna do modelo de Becker (1968), a qual explicita alguns entendimentos ora subentendidos na proposição original, supõe que o indivíduo $j$ é neutro ao risco e racional:

$$
U^{E}=[(1-\text { prisão }) * j]-(\text { prisão } * \text { sentença })-c_{j}-w_{j}-m_{j}
$$

Onde que (1 - prisão) representa a probabilidade de não ser preso, $Y_{j}$ é o ganho da atividade ilícita e, por consequência, $\left[(1-\right.$ prisão $\left.) * Y_{j}\right]$ revela o ganho esperado dessa atividade caso não haja prisão. Por sua vez, caso ocorra a prisão do criminoso o valor esperado da punição será dado por (prisão * sentença). O custo de execução do ato criminoso é $c_{j}$, enquanto $w_{j}$ diz respeito ao custo de oportunidade do crime (rendas advindas das atividades legais) e $m_{j}$ o transgressor.

Portanto, se a utilidade líquida esperada $\left(U^{E}\right)$ for maior do que zero, a racionalidade maximizadora econômica do transgressor o incentivará a cometer a transgressão. As variáveis de deterrence (prisão e sentença) demonstram, respectivamente, para o papel desempenhado pelas forças policiais, pelo judiciário e pelo sistema carcerário. $\mathrm{O}$ custo de execução do crime $\left(c_{j}\right)$ pode então estar inversamente relacionado com a reincidência criminal, uma espécie de especialização no crime, ou até mesmo com a escolaridade, que tornaria o criminoso mais hábil e eficiente. $\mathrm{O}$ custo de oportunidade $\left(w_{j}\right)$ simboliza os ganhos potenciais no mercado de trabalho, influenciados pelos anos médios de estudo ou pela situação econômica. O posicionamento ético-moral acerca do ato de cometer ou não do delito $\left(m_{j}\right)$ tem caráter subjetivo, mas pode estar relacionado, por exemplo, à religiosidade ou à indignação frente a uma situação desigual de distribuição da 
renda (PEREIRA FILHO, 2008).

O modelo econômico do crime teorizado por Becker prevê que o sinal esperado para a relação entre variáveis de deterrence e taxas de crimes seja negativo. No entanto, e possível que os gastos públicos com segurança acompanhem a incidência das taxas de crimes da região (ARAUJO JUNIOR E FAJNZYLBER, 2001; GUTIERREZ ET AL, 2004)

Os modelos econômicos baseados na teoria proposta por Becker (1968) restringe-se ao aspecto financeiro do assunto, o que caracteriza a explicação dos determinantes da criminalidade, incompleta. Ao longo da vida, o individuo busca construir relações que inicialmente nascem no berço familiar e perpassam pelo ambiente de convivência em sociedade. Esse ambiente torna-se fundamental no processo de formação, passando pela construção do julgamento moral e na futura decisão de cometer um crime ou na realização de atividades lícitas.

O objetivo do trabalho de Glaeser e Sacerdote (1999) é construir um modelo que explore a relação da criminalidade e o tamanho das cidades. Segundo o modelo proposto por Becker (1968), tem-se:

$$
B>W+M C+P(P u)
$$

Dado que B representa os benefícios do crime, W o custo de oportunidade, M é o custo moral, $\mathrm{C}$ é custo de execução e planejamento do crime e o termo $\mathrm{P}(\mathrm{Pu})$ representa o custo associado a punição $(\mathrm{Pu})$ e sua respectiva probabilidade de ocorrer P. O modelo assume atributos individuais representados por $\mathrm{X}$, determinados em parte pela trajetória de vida do individuo, que podem influenciar o custo de oportunidade, custo moral, custo de execução e planejamento da ação ilícita e finalmente o custo associado à punição.

As variáveis também são influenciadas pelo ambiente em que os indivíduos estão inseridos, estas influências são representadas por Z. Existem também características de cada cidade, que podem ser o seu tamanho, sua forma de organização, sua forma de lidar com criminosos, suas formas de inserção social, entre outras. Estas características que representam o macrossistema de cada local são representadas por Y. Estas influenciam diretamente os benefícios do crime e a probabilidade de ser punido. Estas características locais também afetam o ambiente em que os indivíduos estão inseridos, pois cada cidade tem um conjunto de características diferentes.

Assumindo que o benefício do crime é uma função decrescente da quantidade de crimes cometidos, denotados por $\mathrm{Q}$, no equilíbrio têm-se que:

$$
B(Y, Q)=W(X, Z(Y))+M(X, Z(Y))+C(X, Z(Y))+P(Y) P u(X, Z(Y))
$$


Diferenciando (2) pode-se determinar como os atributos locais afetam a criminalidade na equação (3) abaixo:

$$
\frac{\partial Q}{\partial Y}=\frac{\left(W_{Z}+M_{Z}+C_{Z}+P . P u_{Z}\right) Z^{\prime}(Y)+\left(W_{X}+M_{X}+C_{X}+P . P u X_{X}\right) \frac{d X}{d Y}+P^{\prime}(Y)(P u)-B_{Y}}{B_{Q}}
$$

Assim, a quantidade média de crimes em uma determinada cidade depende de suas características locais, representadas por Y. As características locais afetam a probabilidade de ser punido, representado pelo termo $\frac{(P(Y) P u}{B_{Q}}$ e alteram o retorno do crime, representado pelo termo $-\frac{B_{Y}}{B_{Q}}$. Como estas características também afetam o ambiente em que os indivíduos estão inseridos, então os custos do crime também são afetados por características endógenas, representados pelo termo: $\frac{\left(W_{Z}+M_{Z}+C_{Z}+P . P u_{Z}\right) Z^{\prime}(Y)}{B_{Q}}$. A quantidade de crimes também depende de atributos individuais exógenos, representados pelo termo: $\frac{\left(W_{X}+M_{X}+C_{X}+P . P u X_{X}\right) \frac{d X}{d Y}}{B_{Q}}$. Este modelo permite discutir a criminalidade em cidades a partir de quatro tipos de explicação: o efeito das características das cidades no retorno do crime e na probabilidade de ser punido; e através de características exógenas e endógenas. A equação (3) acima também pode ser reescrita na forma de elasticidades:

$$
\varepsilon_{Y}^{Q}=\varepsilon_{P}^{Q} \varepsilon_{Y}^{P}-\frac{B_{Y}}{P(Y) P u} \varepsilon_{P}^{Q} \varepsilon_{Y}^{B}+\left(W_{Z}+M_{Z}+C_{Z}+P . P u_{Z}\right) \frac{Z^{\prime}(Y) Y}{B_{Q} Q}+\left(W_{X}+M_{X}+C_{X}+P . P u X_{X}\right) \frac{Y}{B_{Q} Q} \frac{d X}{d Y}
$$

Assim, a elasticidade do crime com relação às características uma cidade depende:

1) Da elasticidade da criminalidade com relação à probabilidade de ser punido; 2) Da elasticidade da probabilidade de ser punido a característica da cidade; 3) Da elasticidade de benefício do crime à característica da cidade; e 4) De características endógenas e exógenas.

Glaeser, Sacerdote, Scheinkman (1996), analisaram a alta variância nas taxas de crime através do espaço, argumentando que há evidência da existência de interações sociais entre os criminosos. O principal resultado do trabalho de Glaeser e Sacerdote (1999) é que as taxas de crime entre as diferentes cidades não necessariamente convergem. Demonstram também que a característica mais importante das populações urbanas na explicação das taxas de crime encontradas nas cidades dos EUA é a percentagem de domicílios chefiados por mulher (FAJNZYLBER E ARAUJO JR., 2000).

\section{EVIDÊNCIAS EMPÍRICAS PARA O BRASIL}

O ponto principal na busca de informações sobre crime e violência para estudos econômicos é por meio da base de dados do Sistema de Informações sobre Mortalidade, 
elaborado e divulgado pelo Ministério da Saúde, os registros da polícia civil e militar, as pesquisas de vitimização, o Anuário Estatístico do Instituto Brasileiro de Geografia e Estatística (IBGE) e os registros do Sistema de Justiça. Essas fontes de dados são apresentadas e discutidas com bastante propriedade nos trabalhos Fajnzylber e Araujo Junior (2001), Soares et al (2003) e Kahn (2000).

A fonte de dados disponível pela Secretaria Nacional de Segurança Pública (SENASP) constitui parte do processo de elaboração de um Sistema Nacional de Estatística de Segurança Pública e Justiça Criminal. Essas estatísticas são elaboradas a partir do número de ocorrências registradas pelas Polícias Civis de todo o Brasil, cujas informações estão disponíveis para os diferentes níveis de agregação: Brasil, regiões geográficas, unidades da federação, capitais e para os cem maiores municípios (SANTOS E KASSOUF, 2008).

Outra fonte de dados útil para analises econômicas da criminalidade é o Sistema Integrado de Informações Criminais do Estado de São Paulo (SIIC) divulgada pela Fundação Sistema Estadual de Analise de Dados (SEADE). Nesse sistema podem ser encontradas informações sobre o funcionamento do sistema judicial de São Paulo, permitindo conhecer, por exemplo, características demográficas e sociais de indivíduos que tem passado pelo sistema desde os indiciados aos condenados. Tais informações contribuem para a escolha de variáveis de controle nos modelos econométricos para modelagem do comportamento criminoso (SANTOS E KASSOUF, 2008).

Há diversas dificuldades empíricas encontradas na investigação econômica sobre crime. As dificuldades mais comuns são: a falta de medida adequada dos retornos da criminalidade, erro de medição nas taxas de crimes em função do elevado número de subregistros e a difícil mensuração da probabilidade de punição (forte correlação com o aumento da criminalidade) (ANDRADE E LISBOA, 2000).

No Brasil, a maior parte da literatura econômico do crime tem se utilizado de dados estaduais e municipais, salvo as exceções de alguns trabalhos realizados com microdados de indivíduos da população carcerária. Nos trabalhos referentes à microdados, podemos enunciar Mendonça (2002), Carneiro et al (2005) e Shikida et al (2006).

No contexto do crime organizado, por exemplo, há forte relação entre o crime organizado e a taxa de homicídios. Santos e Kassouf (2007) encontraram evidências de que o crime organizado, mais especificamente, o mercado de drogas, é um dos responsáveis pelas taxas de homicídios registradas para os estados brasileiros. Carneiro et al (2005), ao analisar uma população de detentos, identificou que um individuo sob o efeito de drogas aumenta a 
probabilidade de cometer um homicídio. As evidências empíricas da relação positiva entre o mercado de drogas e a criminalidade sem drogas são observadas por Donohue e Levitt (1998), Blumstein (1995), Grogger e Willis (2000).

O trabalho de Mendonça (2002) propôs-se a investigar a existência de padrões de comportamento diferenciados entre os indivíduos que praticaram crimes violentos, tendo como base os indivíduos que cometeram crimes não violentos. Os resultados indicam que a idade e o nível educacional do chefe da família são negativamente relacionados com a probabilidade de agir violentamente. Usando o procedimento de Heckman, objetivaram testar a hipótese de que indivíduos ligados a famílias estáveis tendem a praticar menos crimes violentos. Os resultados sustentam a hipótese da diferença entre os indivíduos ligados a crimes violentos dos indivíduos ligados a crimes não violentos. Shikida et al (2006) observaram que a parceria no crime aumenta a probabilidade de o crime ser realizado de forma violenta, diferente do resultado apontado por Mendonça (2002). Nessa mesma linha de discussão, o trabalho de Carneiro et al (2005) envolvendo microdados da população carcerária, observaram que a interação social é determinante do comportamento criminoso. A educação neste contexto se mostrou como um fator de redução na probabilidade de cometer um homicídio intencional, mas fator de incremento na probabilidade de participação no mercado de drogas. Becker (1968) já havia identificado que quanto maior o custo de oportunidade, maior a probabilidade de atuação na atividade ilícita. Além disso, o tráfico de drogas propicia maior probabilidade de atuação nessa área para indivíduos de mais escolaridade.

Os estudos relativos aos determinantes da criminalidade podem ser identificados por meio de modelos empíricos de estimação que visem um dado nível de agregação geográfica, estadual ou municipal. Os trabalhos que utilizam dados municipais nas estimativas de modelos de natureza específica para estudar determinantes da criminalidade podem ser encontrados em Pereira e Fernandez-Carrera (2000), Lobo e Fernandez-Carrera (2003) e Oliveira (2005), Lemos et al (2005), Araújo Junior e Fajnzylber (2000), dos Santos (2010), Cavalcanti et al (2012), Menezes et al (2012), Becker e Kassouf (2012), de Almeida e Guanziroli (2012), Uchoa e Menezes (2012) e Souza, Silveira Neto e Carazza (2012).

O trabalho de Lobo e Fernandez-Carrera (2003) utiliza dados longitudinais referentes a dez municípios da região metropolitana de Salvador. Os dados referem-se a total de crimes, crimes contra o patrimônio, roubo e furto. Os resultados apontam que a probabilidade de detenção atua como efeito deterrence demonstrando o efeito do policiamento. E ainda, 
maiores níveis educacionais, expansão das rendas do município, distribuição de renda podem contribuir para redução nas taxas de criminalidade. No trabalho de Pereira e FernandezCarrera (2000) para a cidade de São Paulo, Os resultados econométricos mostraram que a redução dos índices de desemprego e da concentração de renda, bem como a melhoria no rendimento médio do trabalho, resultantes do crescimento econômico, contribuem para reduzir a atividade criminal.

Uchoa e Menezes (2012) utilizaram dados longitudinais provenientes do Sistema de informações sobre mortalidade (SIM-DATASUS) e correspondem ao número de mortes por agressão, intervenções legais e operações de guerra por 100.000 habitantes, referente aos estados brasileiros, no período de 2005 a 2009 tendo a base de dados composta por um conjunto de 135 observações tomadas no tempo e no espaço. Modelos assim são denominados dados em painel, considerando a heterogeneidade individual. A variável explicativa é a taxa de homicídios, podendo ser vista como uma proxy para os índices de criminalidade. Os resultados demonstram cálculo dos efeitos diretos, indiretos sugerem aumento (ou redução) na criminalidade em um estado afeta positivamente os estados vizinhos. As características socioeconômicas como desigualdade e desemprego, assim como a impunidade e densidade populacional são as variáveis mais importantes para explicar o comportamento das taxas de homicídio entre os estados brasileiros.

Lemos et al (2005), que investigaram a criminalidade com dados de 36 bairros de Aracaju. Fatores que afetam o patrimônio e contra a pessoa foram estudados aplicando regressão do tipo stepwise para selecionar as variáveis explicativas da criminalidade. Variáveis relativas aos moradores do bairro foram incorporadas ao modelo. Os resultados apontam que a concentração de renda, infra-estrutura dos bairros, densidade demográfica e a participação dos jovens no total da população são determinantes da criminalidade. Fator interessante destacado no estudo foi a ocorrência dos crimes em bairros que a faixa etária é mais alta. Isso explica pela proximidade dos bairros, onde ha dependência espacial nas taxas de crimes. È possível que haja deslocamento dos criminosos jovens para bairros mais ricos com o intuito de realizar ato ilícito, evidenciado, por exemplo, no trabalho de Araujo Junior e Fajnzylber (2000), ao estudar a criminalidade nas microrregiões mineiras, porém gerando controvérsia nos resultados, dado que o nível de agregação para bairros é maior em relação a municípios, demonstrando a dependência espacial, como demonstrado por Peixoto (2003) e Almeida et al (2005). 
A criminalidade é potencializada pelo fator tamanho da população residente. Oliveira (2005) utilizando dados longitudinais de todas as cidades brasileiras testou essa hipótese. Os resultados demonstram que o tamanho das cidades é determinante para o aumento da criminalidade. Os autores destacam ainda que o crescimento econômico não afeta diretamente o aumento das taxas de homicídios, desde que ocorra aumento na renda. Os resultados apontam ainda que o aumento da renda dos mais ricos aumenta a criminalidade, gerando efeito contrário aos mais pobres.

As evidencias demonstram que a maior parte das pesquisas é realizada com dados de painel dos estados. Andrade e Lisboa (2000) analisam a relação entre a evolução das taxas de homicídios nos estados de Minas Gerais, Rio de Janeiro e São Paulo entre 1981 e 1997. Esse trabalho busca investigar a violência pela construção de frequências de morte por homicídio para idade, sexo, ano e região de residência. Os resultados demonstram a existência de um "efeito inercial" sobre as taxas de homicídios, de modo que parte da violência vivida por determinada geração tende a se perpetuar para as gerações seguintes. Ainda nos resultados evidenciou-se que os parâmetros estimados para as variáveis econômicas, como salário real e desemprego, são bastante diferenciados dependendo da coorte considerada, sugerindo este método de tratamento de dado como adequado para a investigação da criminalidade.

No Brasil, os estudos empíricos envolvendo determinantes da criminalidade tem utilizado as taxas de homicídios intencionais divulgadas pelo Sistema de Informações sobre Mortalidade, do Ministério da Saúde (SIM), e outras fontes como proxy para a criminalidade como proposto no trabalho de Fajnzylber e Araujo Junior (2001). O trabalho de Becker (1968) relata a existência de uma relação e positiva entre o retorno esperado da atividade ilegal e o crime, de forma inequívoca. Entretanto, ao se utilizar a renda per capita como proxy para os retornos esperados do crime, não se pode afirmar que essa variável estaria associada apenas a ganhos do crime, neste caso, evidenciando uma relação positiva, no entanto poderia haver relação negativa associada a custos de oportunidade.

Os modelos econométricos empregam variáveis explicativas: O emprego de outras variáveis de deterrence alternadamente; inclusão de medidas alternativas de desigualdade de renda, assim como a percentagem da população com renda abaixo de duas linhas diferentes de pobreza; e uma investigação da relação existente entre a taxa de homicídios e diferentes medidas de mobilidade social. 
Fajnzylber e Araujo Junior (2001) testaram a robustez dos resultados na presença de endogeneidade nas variáveis explicativas pela aplicação do Método Generalizado de Momentos (GMM), sugerido por Arellano e Bond (1991), por meio do qual é possível investigar a existência de inércia nas taxas de crimes, utilizando a taxa de homicídios defasada em um período como variável explicativa do modelo. Os resultados indicam que os parâmetros estimados para a renda per capita, desemprego, desigualdade de renda e percentagem de domicílios chefiados por mulheres foram estatisticamente positivos, enquanto que o quantitativo de policiais militares por cem mil habitantes se mostrou negativamente relacionado as taxas de crimes.

Fajnzylber e Araujo Junior (2001) investigam também a existência de ciclos de vida nas taxas de homicídios dos estados brasileiros utilizando variáveis econômicas para explicalos, por uma metodologia de decomposição das taxas de crimes em efeitos idade (ciclo de vida), período com choques temporários e coorte, conforme proposto na literatura por Deaton (1997). Os resultados encontrados sugerem que o ambiente econômico seria parcialmente responsável pela criminalidade observada no Brasil no período entre 1981 e 1996 e que, em alguma medida, o efeito das variáveis econômicas é diferenciado dependendo da faixa etária considerada.

Nos estudos que utilizam dados em painel, as técnicas utilizadas mostram-se mais apropriadas no caso dos estudos econômicos envolvendo a teoria do crime, por permitir o controle da heterogeneidade não-observável existente entre as unidades individuais, permitindo também o controle parcial do problema de erro de medida decorrente da alta taxa de sub-registro de crimes.

Gutierrez et alii (2004) utilizam dados agregados dos estados brasileiros cobrindo o período de 1981 a 1995 para investigar os determinantes econômicos do crime, utilizando como proxy a taxa de homicídios intencionais por 100 mil habitantes. Os resultados sugerem que a taxa de desemprego, a urbanização e a desigualdade de renda exercem inequivocamente efeitos positivos sobre a taxa de homicídios. A desigualdade de renda parece estar relacionada á taxa de homicídios no Brasil (MENDONÇA, 2002) ao propor e testar uma extensão do modelo de Becker num contexto de otimização intertemporal. Utilizando, o estimador system GMM, conforme proposto por Blundell e Bond (1998), confirmam-se os resultados de Andrade e Lisboa (2000), Araujo Junior e Fajnzylber (2001) e Fajnzylber e Araujo Junior (2001), Kume (2004) e Santos (2010) no que diz respeito à presença de inércia nas taxas de homicídios. Santos (2010) aprofundaram ainda mais a 
discussão a respeito do efeito dissuasório dos gastos com segurança pública, não sendo observado. Porém, aumentar a escolaridade é uma das formas de reduzir a taxa de crimes letais nos estados brasileiros.

Becker e Kassouf (2012), realizaram trabalho que buscava evidências da relação entre os gastos públicos com educação e o crime através do estimador System GMM com defasagens para tais gastos. A metodologia proposta consistia na estruturação de painel de dados composto pelos 26 estados brasileiros e o Distrito Federal, observados entre os anos de 2001 a 2009, dessa forma, a amostra é composta por 243 observações. Conforme Santos e Kassouf (2007) a estrutura de dados em painel é a melhor estratégia para lidar com os problemas das estimativas de criminalidade, que geralmente apresentam heterogeneidade não observável entre as unidades individuais, no caso os Estados, e também apresentam erro de medida. A heterogeneidade está relacionada às características culturais que influenciam de forma diferenciada as taxas de crimes nos Estados, como por exemplo, o consumo de drogas e álcool, a presença de atividades ilegais particularmente lucrativas, etc. Já o erro de medida é decorrente da taxa de sub-registro de crimes. As estimativas foram feitas através dos estimadores Generalized Method of Moments - GMM (ARELLANO E BOND, 1991) e System GMM (ARELLANO E BOVER 1995; BLUNDELL E BOND, 1998). Os resultados desta pesquisa sugerem que políticas públicas voltadas para a educação podem contribuir para reduzir a criminalidade.

No Brasil, a literatura que investiga as peculiaridades causadas pelo emprego da análise espacial sobre a criminalidade na abordagem econômica aparece no trabalho de Sartoris Neto (2000), que realizou uma analise de autocorreção e causalidade espaçotemporal das taxas de homicídios na cidade de São Paulo.

No trabalho de Peixoto (2003), ao utilizar dados de registros policiais das Unidades de Planejamento do município de Belo Horizonte, investigou os determinantes socioeconômicos da criminalidade. A analise enfocou os crimes violentos contra o patrimônio e crimes violentos contra a pessoa. Foram empregadas as técnicas exploratória espacial, que permite identificar clusters criminais e regiões atípicas e também a presença de autocorrelação e heterogeneidade espacial, e de econometria espacial que investiga peculiaridades espaciais consideradas dentro do modelo estocástico.

Os resultados importantes do estudo são que a taxa de homicídios no município de Belo Horizonte é concentrada em algumas regiões, constatando também que nas periferias e favelas em geral, as taxas de roubos são relativamente baixas comparadas 
as demais regiões, entretanto a taxa de homicídios é relativamente alta na periferia. Também revelaram que o nível de riqueza e o tempo médio de atendimento da polícia exercem, respectivamente, efeitos negativos e positivos sobre a taxa de homicídios. Desta forma, percebe-se que há dependência espacial nos dados das Unidades de Planejamento do município de Belo Horizonte.

Almeida et alii (2005), na mesma linha de investigação, ao utilizar a taxa de homicídios intencionais nos municípios do estado de Minas Gerais, investigam o padrão espacial da criminalidade, confirma os resultados encontrados por Peixoto (2003).

Dos santos e dos Santos filho (2011), analisaram a hipótese da existência de taxa de convergência da criminalidade ao longo dos anos para o território brasileiro, utilizando a taxa de homicídios como proxy para a criminalidade e controle da dependência espacial para dados regionalmente agrupados por microrregiões. Os resultados apontam que a convergência nas taxas de crimes intra-regionais significa que a criminalidade tende a crescer mais rapidamente nas localidades menos violentas do que nas mais violentas. Desta forma, a diferença nas taxas de crimes observadas entre essas localidades será gradativamente eliminada ao longo do tempo.

De almeida e Guanziroli (2012) analisam os determinantes dos crimes violentos contra o patrimônio e contra a vida do individuo para Minas Gerais usando dados longitudinais de 2000 a 2007. Os crimes violentos contra o patrimônio foram mais bem estimados pelo modelo de efeito fixo, enquanto os homicídios obtiveram melhor resposta quando regredidos por efeito aleatório, ambos considerando a endogeneidade. Como os municípios foram tomados como unidades espaciais consideraram-se a presença da associação espacial dos crimes sob o controle de dummies construídas a partir do cálculo da Autocorrelação espacial local do crime. Em análise logarítmica, a taxa de detenção de crimes violentos possui relação negativa sobre esses delitos, assim como o armamento da população. Por outro lado, a renda media do trabalho formal aumenta o quantitativo de crimes contra o patrimônio.

Em relação aos crimes contra a vida, os testes de Hausman e de Breush Pagan revelaram que a melhor opção é regredir o modelo por efeito aleatório. Por outro lado, outros fatores como o montante de armas em posse da população, o acesso dos jovens ao ensino médio sem atraso escolar, o envolvimento social e valores morais, assim como o controle espacial, revelaram-se importantes para explicar os homicídios. Os determinantes dos crimes contra vida foram estimados por GMM acrescentando um indicador de desenvolvimento econômico, o Indice Firjan de Desenvolvimento Municipal. Esse fator revelou-se forte e 
negativamente relacionado aos homicídios, mostrando que o desenvolvimento econômico teria efeitos negativos sobre esse tipo de crime. Portanto, para que haja reduções nas mortes causadas por violência intencional, as autoridades promover o desenvolvimento econômico, sobretudo na educação, com atenção especial aos adolescentes, equipando e qualificando suas forcas de policiais.

Cavalcanti et al (2012) investigaram a influência de características socioeconômicas dos municípios brasileiros sobre a redução das taxas de mortalidade infantil, com controle de spillovers espaciais. A base de dados foi o censo de 1991 e 2000, tendo como unidade de observação as áreas mínimas comparáveis definidas pela agência de dados do Brasil, IBGE. Em seguida, realizou-se análise exploratória de dados espaciais. Foram calculados os efeitos diretos, indiretos e o efeito total das características sócioeconômicas sobre a variável dependente. Os resultados apontaram que as maiores reduções nas taxas de mortalidade infantil ocorreram em áreas com maiores taxas de mortalidade defasadas, sugerindo a presença de convergência condicional e que a estimativa da taxa de analfabetismo indicou que as taxas mais elevadas de analfabetismo estão associadas com menor declínio nas taxas de mortalidade infantil, sugerindo indícios de pobreza. Também, verificou-se que a melhoria da educação dos próprios municípios, atuando em saneamento e urbanização nos municípios vizinhos, são determinante das mudanças nas taxas de mortalidade infantil.

Menezes et al (2012), buscaram investigar o padrão espacial dos homicídios nos bairros de Recife, Trabalhando com uma base de dados para Recife entre os anos de 2008 e 2010, com a intenção de capturar a dependência espacial nas taxas de homicídio. Para isto foram calculados os efeitos diretos, indiretos e totais, seguindo a metodologia proposta por LeSage and Pace ( 2009) e Elhorst (2011) das características dos bairros sobre os homicídios. A variável dependente neste estudo foi a taxa média de homicídio de homens por 100.000 habitantes nos anos de 2008, 2009 e 2010. O Homicídio foi definido como os crime violento intencional que resulta em morte. A taxa de homicídio foi calculada de acordo com o número de crimes para os anos referencia, usando os dados de população do Censo 2010. A fim de contornar a endogeneidade, foram usadas variáveis defasadas para o ano de 2000. Os dados sobre homicídios foram cedidos pela Secretaria de Defesa Social do Estado de Pernambuco ao nível dos bairros. Os resultados encontrados foram, após exercitar um controle para renda e desigualdade, as áreas com maiores taxas de homicídio foram aquelas com as maiores populações e aquelas com maior crescimento da densidade populacional. Através do controle de outras variáveis, nota-se que a desigualdade possui efeito positivo e significante na 
criminalidade. No entanto, esta influência é mitigada pela natureza da dependência espacial da criminalidade entre os bairros.

\section{CONSIDERAÇÕES FINAIS}

Um dos fatores que certamente contribuem para o crescimento da criminalidade e a alta taxa de reincidência criminal. Há carências de evidências para o Brasil para esse tipo de investigação (SANTOS E KASSOUF, 2008). Outro fator que poderia ser levado em consideração para novos estudos empíricos diz respeito ao fator de ciclo de vida do criminoso, tema este abordado por Pezzin (1994) para os Estados Unidos.

A literatura da área demonstra que a produção científica é importante para compreensão e investigação, visando melhorias nas políticas públicas, principalmente relacionadas ao policiamento e suas deficiências estruturais, bem como em relação as oportunidades e condições de vida ao individuo.

O que se pode observar é que as pesquisas possuem objetivos específicos quando a determinação das causas da criminalidade. Há também indícios de controvérsias quanto as variáveis de deterrence. No entanto, observa-se consenso quanto a desigualdade de renda que afeta o comportamento criminoso. Existem indícios de que a criminalidade é afetada por circunstâncias espaciais. Os principais problemas relacionados a pesquisa devem-se principalmente à carência de dados relacionados a crime. A maioria dos estudos utiliza taxas de homicídios de forma intencional para tentar medir a criminalidade.

\section{REFERÊNCIAS}

ALMEIDA, E. S.; HADDAD, E. A.; HEWINGS, G. J. D. (2005). The spatial pattern of crime in Minas Gerais : An explanatory analysis. Economia Aplicada, 9(1):39-55.

ANDRADE, M. V.; LISBOA, M. B. (2000). Desesperança de vida: Homicídio em Minas Gerais. In: Henriques, R., editor, Desigualdade e Pobreza no Brasil, p. 347-384. IPEA, Rio de Janeiro.

ARAÚJO JR., A. F. DE; FAZNZYLBER, P. (2000) Crime e economia: um estudo das microrregiões mineiras. Revista Econômica do Nordeste. v. 31, número especial, novembro de 2000. p.630-659.

ARELLANO, M.; BOND, S. (1991). Some tests of specification for panel data: Monte Carlo evidence and application to employment equations. Review of Economic Studies, 58:277-297.

ARELLANO, M.; BOVER, O. (1995). Another look at the instrumental variable estimation of error-components models. Journal of Econometrics, 68:29-51. 
BECKER, G. S. (1968) Crime and Punishment: An Economic Approach. The Journal of Political Economy. v.76, n.2, p. 169-217, mar.-abr. 1968.

BECKER, K. L.; KASSOUF, A. L. (2012) Impacto dos gastos com educação sobre a criminalidade no brasil. In: Anais do $\mathbf{X}$ encontro nacional da associação brasileira de estudos regionais e urbanos - ENABER. Recife/PE, 2012.

BLOCK, M. K.; HEINECKE, J. M. (1975) A Labor Theoretic Analysis of the Criminal Choice. American Economic Review. v.65, p.314-325, 1975.

BLUNDELL, R.; BOND, S. (1998). Initial conditions and moment restrictions in dynamic panel data models. Journal of Econometrics, 87:115-143.

BLUMSTEIN, A. (1995). Youth violence, guns and the illicit-drug industry. Journal of Criminal Law and Criminology, 86(1):10-36.

BRUECKNER, J. K. (2011) Lectures on urban economics. MIT Press Books, v. 1, 2011.

CARNEIRO, F. G.; LOUREIRO, P. R. A.; SACHSIDA, A. (2005). Crime and social interactions: A developing country case study. The Journal Socio-Economics, 34:311318.

CARVAlho, A. X.; CERQUEIRA, D. R. C., RODRIGUES, R. I., \& LOBAO, W. J. A. (2007). Custos das mortes por causas externas no Brasil. Brasília, IPEA, 33p. T.D. 1268.

CAVAlCANTI, E. M.; UCHOA, C. A.; CASTRO E SILVA, T. B.; DE MENEZES, T. A. (2012) Empirical evidences and analysis of spillovers over infant mortality rate in brazil between 1991 and 2000 In: Anais do X encontro nacional da associação brasileira de estudos regionais e urbanos - ENABER. Recife/PE, 2012.

CERQUEIRA, D.; LOBÃO, W. (2003) Determinantes da Criminalidade: Uma Resenha dos Modelos Teóricos e Resultados Empíricos. Brasília: Instituto de Pesquisa Econômica Aplicada, jun. 2003. (Texto para Discussão nº 956).

CERQUEIRA, D. R. C.; CARVALHO, A.X.Y; LOBÃO, W.J.A.; RODRIGUES, R.I. (2007) Análise dos Custos e das Consequências da Violência no Brasil . Brasília: Instituto de Pesquisa Econômica Aplicada, jun. 2007. (Texto para Discussão no 1284).

CORNWELL, C.; TRUMBULL, W. N. (1994) Estimating the Economic Model of Crime with Panel Data. The Review of Economics and Statistics. v.76, n.2, p. 360-366, maio 1994.

CORMAN, H. \& MOCAN, H. N. (2000). A time-series analysis of crime, deterrence and drug abuse in New York city. The American Economic Review, 90(3):584-604.

CRAIG, S. G. (1987) The Deterrent Impact of Police: An Examination of a Locally Provided Public Service. Journal of Urban Economics. v.21, p.298-311, 1987.

DE MENDONÇA, M. J. C. (2001) Um modelo de criminalidade para o caso brasileiro. In: Anais do XXIX Encontro Nacional de Economia [Proceedings of the 29th Brazilian Economics Meeting]. ANPEC-Associação Nacional dos Centros de Pósgraduação em Economia [Brazilian Association of Graduate Programs in Economics], 2001. Disponível em: < http://www.anpec.org.br/encontro2001/artigos/200106193.pdf>. Acesso em: 10.07.2013.

DE ALMEIDA, M. A. S.; GUANZIROLI C. E. (2012) Fatores explicativos da criminalidade mineira: estimativas a partir do painel de dados de 2000 a 2007, considerando efeitos espaciais. In: Anais do $\mathrm{X}$ encontro nacional da associação brasileira de estudos regionais 
e urbanos - ENABER. Recife/PE, 2012.

DOS SANTOS, M. J.(2010). Dinâmica temporal da criminalidade: Mais evidências sobre o "efeito inércia" nas taxas de crimes letais nos estados brasileiros. Revista Economia, 2009.

DOS SANTOS, M. J.; DOS SANTOS FILHO, J. I. (2011) Convergência das taxas de crimes no território brasileiro. Revista EconomiA,

DEATON, A. (1997). The Analysis of Holsehold Surveys: A Microeconomic Approach to Development Policy. Johns Hopkins University Press, Baltimore, Maryland.

DONOHUE III, J. J.; LEVITT, S. D. (1998). Guns, violence and the efficiency of illegal markets. The American Economic Review, 88(2):463-467.

DONOHUE, J.; LEVITT, S. (2001) The Impact of Legalized Abortion on Crime. Quarterly Journal of Economics. v.116, n.2, p. 379-420, 2001.

EHRLICH, I. (1973) Participation in Illegitimate Activities: A Theoretical and Empirical Investigation. The Journal of Political Economy. v.81, n.3, p. 521-565, maio-jun. 1973.

ELHORST, J. P. (2011). Dynamic spatial panels: models, methods, and inferences. Journal of Geographical Systems. Volume 14, Number 1, pp. 5-28, September.

FAJNZYLBER, P.; ARAUJO JUNIOR, A. F. (2001). Violência e criminalidade. In Lisboa, M. B. \& Menezes Filho, N. A., editores, Microeconomia e Sociedade no Brasil, p. 333394. Contra Capa, Rio de Janeiro.

FAJNZYLBER, P., LEDERMAN, D., LOAYZA, N. (1998) Determinants of Crime Rates in Latin America and the World: An Empirical Assessment. Washington, DC: World Bank, World Bank Latin American and Caribbean Studies, 1998.

FLEISHER, B. M.(1963) The Effect of Unemployment on Juvenile Delinquency. The Journal of Political Economy. v.71, n.6, p.543-555, dez.1963.

(1966) The Effect of Income on Delinquency. The American Economic Review. v.61, n.1, p.118-137, mar. 1966.

GLAESER, E., SACERDOTE, B., SCHEINKMAN, J. (1996) Crime and Social Interactions. Quarterly Journal of Economics, v. 111, p. 507-548, 1996.

(1999). Why is there crime in cities? Journal of Political Economy, 107(6):225-258

GOULD, E. D.; WEINBERG, B. A.; MUSTARD, D. B. (2000) Crime Rates and Local Labor Market Opportunities in the United States: 1979-1997. JEL Codes, K 4, J 0, p.158 .

GROGGER, J.; WILLIS, M. (2000). The emergence of crack cocaine and the rise in urban crime rates. The Review of Economics and Statistics, 82(4):519-529.

GUTIERREZ, M. B. S.; MENDONÇA, M. J. C.; SACHSIDA, A.; LOUREIRO, P. R. A. (2004). Inequality and criminality revisited: Further evidence from Brazil. In XXXII Encontro Nacional de Economia. ANPEC, João Pessoa. Disponível em: http://www.anpec.org.br/encontro2004/artigos/A04A149.pdf Acesso em 30 de junho de 2013.

KAHN, T. (2000). Os custos da violência: Quanto se gasta ou deixa de ganhar por causa do crime no Estado de São Paulo. Fórum de Debates, Rio de Janeiro: IPEA, CESeC. 
HARTUNG, G., PESSOA, S. Fatores Demográficos como Determinantes da Criminalidade. In: XXXV Encontro Nacional de Economia, Recife, 2007. Disponível em: http://www.anpec.org.br/encontro2007/artigos/A07A112.pdf . Acesso em: 20 junho. 2013.

KUME, L. (2004) Uma Estimativa dos Determinantes da Taxa de Criminalidade Brasileira: Uma aplicação em Painel Dinâmico. In: XXXII Encontro Nacional de Economia, João Pessoa, 2004. Disponível em: http://econpapers.repec.org/paper/anpen2004/148.htm. Acesso em: 07 junho. 2013.

LEMOS, A. A. M.; SANTOS FILHO, E. P.; JORGE, M. A. (2005). Um modelo para análise socioeconômica da criminalidade no município de Aracajú. Estudos econômicos, 5(3):569-594.

LESAGE, J. P.; PACE, R. K. (2009) Introduction to Spatial Econometrics. CRC Press, Boca Raton, FL.

LEVITT, S. D. (1997). Using electoral cycles in police hiring to estimate the effect of police on crime. The American Economic Review, 87(3):270-290.

LEVITT, S. D. (1998). Juvenile crime and punishment. Journal of Political Economy, 106(2):1156-1185.

LEVITT, S. (2004) Understanding Why Crime Fell in the 1990s: Four Factors That Explain the Decline and Six That Do Not. Journal of Economic Perspectives. v.18, n.1, p. 163-90, 2004.

LOBO, L. F.; FERNANDEZ-CARRERA, J. (2003). A criminalidade na região metropolitana de Salvador. In XXXI Encontro Nacional de Economia. ANPEC, Porto Seguro. Disponível em: http://econpapers.repec.org/paper/anpen2003/d26.htm. Acesso em 28 de junho de 2013.

LOUREIRO, A. O. F.; CARVALHO JÚNIOR, J. R. A. (2007). O Impacto dos Gastos Públicos Sobre a Criminalidade no Brasil. In XXXV Encontro Nacional de Economia, Recife, 2007. Disponível em: http://www.anpec.org.br/encontro2007/artigos/A07A163.pdf. Acesso em: 15 junho. 2013.

MENDONCA, M. J. C. (2002). Criminalidade e violência no Brasil: Uma abordagem teórica e empírica. Revista Brasileira de Economia de Empresas, 2(1):33-49.

MENEZES, T; SILVEIRA NETO, R. M; MONTEIRO, C.; RATTON, J. L.; (2012) Spatial correlation between homicide rates and inequality: evidence from urban neighborhoods in a brazilian city. In: VI World Conference - Spatial Econometrics Association - SEA. Salvador, Brazil, 2012.

OLIVEIRA, C. A. (2005) Criminalidade e o Tamanho das Cidades Brasileiras: Um Enfoque da Economia do Crime. In: XXXIII Encontro Nacional de Economia, Natal, 2005. Disponível em: http://econpapers.repec.org/paper/anpen2005/152.htm. Acesso em: 07 junho 2013.

PEREIRA FILHO, O.A. (2008) Medidas de custo-eficiência nos serviços subnacionais de segurança pública: uma abordagem com o uso de fronteiras estocásticas. $68 \mathrm{f}$. Dissertação. (Mestrado em Economia do Setor Público). Universidade de Brasília, Brasília. 2008.

PEIXOTO, B. T. (2003). Determinantes da criminalidade no município de Belo Horizonte. dissertação de mestrado, Universidade Federal de Minas Gerais, CEDEPLAR, Belo Horizonte. 
PEREIRA, R.; FERNANDEZ-CARRERA, J. (2000). A criminalidade na região policial da grande São Paulo sob a ótica da economia do crime. Revista Economica do Nordeste, 31:898-918.

PEZZIN, L. E. (1994). Incentivos de mercado e comportamento criminoso: Uma analise econômica dinâmica. Estudos Economicos, 24(3):373-404.

SANTOS, M. J.; KASSOUF, A. L (2007). Uma Investigação Econômica da Influência do Mercado de Drogas Ilícitas sobre a Criminalidade Brasileira. Revista EconomiA. Brasília, v.8, n.2, p. 187-221, maio/ago. 2007. Disponível em: <http://www.anpec.org.br/revista/vol8/vol8n2p187_210.pdf>. Acesso em: 05 junho. 2013.

(2008). Estudos Econômicos das Causas da Criminalidade no Brasil: Evidências e Controvérsias. Revista EconomiA. Brasília, 2008 (Artigos Aprovados). Disponível em: $<$ http://www.anpec.org.br/revista/aprovados/Economia_crime.pdf>. Acesso em: 05 de junho 2013.

SARTORIS NETO, A. (2000). Homicídios na Cidade de São Paulo: Uma Análise de Autocorrelação e Causalidade Espaco-Temporal. Tese de doutorado, Faculdade de Economia, Administração e Contabilidade, Universidade de São Paulo.

SOARES, G. A. D.; MUSUMECI, L.; BORGES, D.; RODRIGUES, S. C.; FRAGA, G. (2003). Base nacional de estatísticas criminais: Analise e avaliação. Produto 2 Recomendações de detalhamento e aperfeiçoamento da base de dados. Rio de Janeiro: Universidade Candido Mendes, Centro de Estudos de Segurança Pública, 37p. (relatório de consultoria prestada à Secretaria Nacional de Segurança Pública do Ministério da Justiça.

SHIKIDA, P. F. A.; ARAUJO JUNIOR, A. F.; SHIKIDA, C. D.; BORILli, S. P. (2006). Determinantes do comportamento criminoso: Um estudo econométrico nas penitenciarias central, estadual e feminina de Piraquara (Paraná). Pesquisa e Debate, 17(1):125-148.

SJOQUIST, D. L.(1973). Property Crime and Economic Behavior: Some Empirical Results. The American Economic Review. v.63, n.3, p.439-446, jun. 1973.

TRUMBULL, W. N. (1989). Estimations of the Economic Model of Crime Using Aggregate and Individual Level Data. Southern Economic Journal. v.56, p.423-439, 1989.

UCHOA, C. F. A.; DE MENEZES, T. A. (2012). Spillover espacial da criminalidade: uma aplicação de painel espacial para os estados brasileiros. In XL Encontro Nacional de Economia, Porto de Galinhas/PE, 2012.

WOLPIN, K. (1978). An Economic Analysis of Crime and Punishment in England and Wales, 1894-1967. Journal of Political Economy. v.86, p.815-840, 1978.

. (1980) A Time-Series-Cross Section Analysis of International Variation in Crime and Punishment. The Review of Economics and Statistics. vol.62, p.417-423, 1980.

ZHANG, J. (1997) The Effects of Welfare Programs on Criminal Behavior: A Theoretical and Empirical Analysis. Economic Inquiry. v.35, p.120-137, 1997. 